University of Nebraska - Lincoln

DigitalCommons@University of Nebraska - Lincoln

Timothy J. Gay Publications

Research Papers in Physics and Astronomy

October 1987

\title{
Angular-differential studies of excitation in quasi-oneelectron collisions at "high" energy
}

\author{
E. Redd \\ University of Missouri-Rolla, Rolla, Missouri \\ Timothy J. Gay \\ University of Nebraska - Lincoln, tgay1@unl.edu \\ D. M. Blakenship \\ University of Missouri-Rolla, Rolla, Missouri \\ J. T. Park \\ University of Missouri-Rolla, Rolla, Missouri \\ J. L. Peacher \\ University of Missouri-Rolla, Rolla, Missouri \\ See next page for additional authors
}

Follow this and additional works at: https://digitalcommons.unl.edu/physicsgay

Part of the Physics Commons

Redd, E.; Gay, Timothy J. ; Blakenship, D. M.; Park, J. T.; Peacher, J. L.; and Seely, D. G., "Angular-differential studies of excitation in quasi-oneelectron collisions at "high" energy" (1987). Timothy J. Gay Publications. 14.

https://digitalcommons.unl.edu/physicsgay/14

This Article is brought to you for free and open access by the Research Papers in Physics and Astronomy at DigitalCommons@University of Nebraska - Lincoln. It has been accepted for inclusion in Timothy J. Gay Publications by an authorized administrator of DigitalCommons@University of Nebraska - Lincoln. 


\section{Authors}

E. Redd, Timothy J. Gay, D. M. Blakenship, J. T. Park, J. L. Peacher, and D. G. Seely 
Phys. Rev. A 36, 3475 - 3478 (1987)

[Issue 7 - October 1987]

\section{Angular-differential studies of excitation in quasi-one- electron collisions at "high" energy}

E. Redd *, T. J. Gay, D. M. Blankenship, J. T. Park, J. L. Peacher, and D. G. Seely Laboratory of Atomic and Molecular Physics and the Physics Department, University of Missouri-Rolla, Rolla, Missouri 65401

Qualitative differences have been observed between two types of "quasi-one-electron" collision systems. We have studied valence-electron excitation at "high" energy (relative collision velocities up to 0.5 a.u.) in the $\mathrm{Mg}^{+}+\mathrm{He}$ and $\mathrm{Na}^{+}+\mathrm{H}$ collision systems, and find that while $\mathrm{Mg}^{+}+\mathrm{He}$ collisions are dominated by "direct" excitation, the $\mathrm{Na}^{+}+\mathrm{H}$ collisions exhibit significant "molecular" excitation, even at the highest velocities. This behavior can be understood in terms of the molecular structure of the respective collision complexes, and the energy separation between the ground and first excited states of the valence electron.

(C)1987 The American Physical Society

URL: http://link.aps.org/abstract/PRA/v36/p3475

DOI: 10.1103/PhysRevA.36.3475 


\title{
Rapid Communications
}

The Rapid Communications section is intended for the accelerated publication of important new results. Since manuscripts submitted to this section are given priority treatment both in the editorial office and in production, authors should explain in their submittal letter why the work justifies this special handling. A Rapid Communication should be no longer than 31/2 printed pages and must be accompanied by an abstract. Page proofs are sent to authors, but, because of the accelerated schedule, publication is not delayed for receipt of corrections unless requested by the author or noted by the editor.

\section{Angular-differential studies of excitation in quasi-one-electron collisions at "high" energy}

\author{
E. Redd, ${ }^{*}$ T. J. Gay, D. M. Blankenship, J. T. Park, J. L. Peacher, and D. G. Seely \\ Laboratory of Atomic and Molecular Physics and the Physics Department, \\ University of Missouri-Rolla, Rolla, Missouri 65401
}

(Received 4 May 1987)

\begin{abstract}
Qualitative differences have been observed between two types of "quasi-one-electron" collision systems. We have studied valence-electron excitation at "high" energy (relative collision velocities up to 0.5 a.u.) in the $\mathrm{Mg}^{+}+\mathrm{He}$ and $\mathrm{Na}^{+}+\mathrm{H}$ collision systems, and find that while $\mathrm{Mg}^{+}+\mathrm{He}$ collisions are dominated by "direct" excitation, the $\mathrm{Na}^{+}+\mathrm{H}$ collisions exhibit significant "molecular" excitation, even at the highest velocities. This behavior can be understood in terms of the molecular structure of the respective collision complexes, and the energy separation between the ground and first excited states of the valence electron.
\end{abstract}

Quasi-one-electron (QOE) collision systems involve an outer "active" electron and either two closed-shell cores or one closed shell and a bare nucleus. The relatively simple nature of such systems makes them an obvious choice for study in order to learn about fundamental collision processes. ${ }^{1}$ A standard view that has developed in the last decade is that excitation of the active electron in such collisions results from either (i) violent collisions involving significant core penetration, in which excitation takes place at well-localized quasimolecular curve crossings or (ii) glancing collisions, where an impulsive, delocalized Coulomb interaction between the valence electron and the closed core is responsible for the excitation. In the former case, the excitation is a many-electron process, whereas the latter case may be regarded essentially as a oneelectron process. These mechanisms are often called "molecular" and "direct" excitation, respectively. While it is in some sense artificial to make distinctions in this manner, the differentiation of the two mechanisms is generally useful in that they lead to significantly different behavior of the excitation probability as a function of impact parameter. Specifically, one would expect molecular excitation to be sensitive to the details of the quasimolecular structure, and thus be strongly impact parameter dependent, while direct excitation should be relatively insensitive to impact parameter.

A class of QOE systems that has been studied extensively is that involving rare-gas targets and neutral alkalimetal or alkaline-earth ion projectiles. ${ }^{1}$ In these systems, molecular excitation dominates at "low" energy (projectile velocities $\lesssim 0.1$ a.u.) and is responsible for a first local maximum or plateau often seen in the total excitation cross section versus $E$. At higher energy, direct excitation dominates, and is characterized by a large, broad maximum (the "Massey peak") in the cross section at a velocity $v$ scaling roughly as $a \Delta E$, where $\Delta E$ is the excitation energy, and $a$ is a somewhat ambiguous interaction length, typically set equal to the combined radii of the two cores. ${ }^{1,2}$

While total excitation cross sections for such collisions have been measured for projectile velocities up to $\simeq 0.5$ a.u. (Refs. 1, 3, and 4), angular differential cross sections, which provide information about the impact parameter dependence of excitation probability, have, until now, been limited to velocities $\leq 0.25$ a.u. ${ }^{1,5}$ Theoretical calculations to date have almost exclusively used the coupled-channel impact parameter approximation, and have enjoyed varying degrees of success. ${ }^{1,6-9}$ Treatments employing molecular basis sets are more appropriate for descriptions of molecular excitation, and are thus most applicable at low energy. Conversely, atomic basis sets should give better results at high energy, where direct excitation is dominant. Unfortunately, even though the excitation mechanism is clearcut in this region, this is exactly where reliable close-coupling (CC) calculations are the most difficult, because of the comparable importance of a large number of reaction channels. Indeed, the agreement between measured total cross sections and theoretical predictions has been, at the higher velocities, quantitative only for isolated cases involving neutral alkali metals incident on He targets. ${ }^{9}$ Significantly, theoretical predictions of total cross sections employing atomic orbital (AO) bases are very sensitive to the way in which the difficult outer-electron- (neutral) rare-gas interaction is handled. ${ }^{8}$

In order to obtain more detailed information about 
QOE collisions in the intermediate velocity regime, we have measured angular differential cross sections (ADCS's) at "high" energy (0.22 a.u. $\leq v \leq 0.5$ a.u.) for two systems: $\mathrm{Mg}^{+}(3 s)+\mathrm{He} \rightarrow \mathrm{Mg}^{+}(\theta, 3 p)+\mathrm{He}$ and $\mathrm{Na}^{+}+\mathrm{H}(n=1) \rightarrow \mathrm{Na}^{+}(\theta)+\mathrm{H}(n=2)$. These ADCS's data are thus the first for QOE systems at projectile velocities above 0.25 a.u. The $\mathrm{Na}^{+}{ }_{-} \mathrm{H}$ system, which has not been studied before, was chosen because it introduces the important simplification of an outer electron-ion interaction. Unfortunately, such collisions are complicated by non-negligible charge-transfer channels, with the attendant theoretical difficulties of electron translational factors. (We note that extensive studies have been made of the $\mathrm{H}^{+}+\mathrm{Na}$ charge-transfer process. ${ }^{10,11}$ )

The cross sections reported in this paper were measured using the technique of ion energy-loss spectroscopy. This technique has the important advantage that data analysis does not require cascade corrections nor the knowledge of detector efficiencies, as do optical measurements. The apparatus we used has been described extensively. ${ }^{12-14}$ Great care was taken in determining the density-thickness product $n l$ of the target. ${ }^{14}$ For $\mathrm{H}$, both a thermal transpiration and normalization method were used, and agreed well with each other. The error in $n l$ is $7.8 \%$ and $3.6 \%$ for $\mathrm{H}$ and $\mathrm{He}$, respectively.

The resulting ADCS's are shown in Figs. 1 and 2. Fundamental differences between the two systems are obvious immediately. First, while the $\mathrm{MgHe}^{+}$cross sections at all energies decrease monotonically with angle, the lowestenergy $\mathrm{NaH}^{+}$ADCS's have a local maximum at -12 mrad in the center-of-mass frame. There appear to be remnants of this structure at 51.8 and $63.9 \mathrm{keV}$. Second, the $\mathrm{MgHe}^{+}$cross sections are roughly an order of magni-

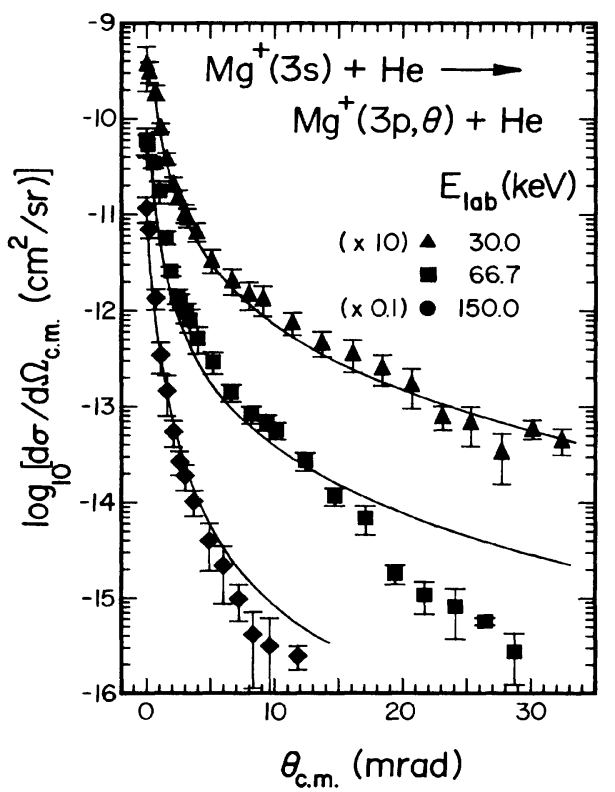

FIG. 1. $\mathrm{Mg}^{+}+\mathrm{He}$ ADCS's. Theory curves ( - ) of Nielsen and Dahler (Ref. 19). Error bars represent the standard deviation of the mean (SDM) of all runs, combined in quadrature with target thickness and solid-angle errors. Note scale changes.

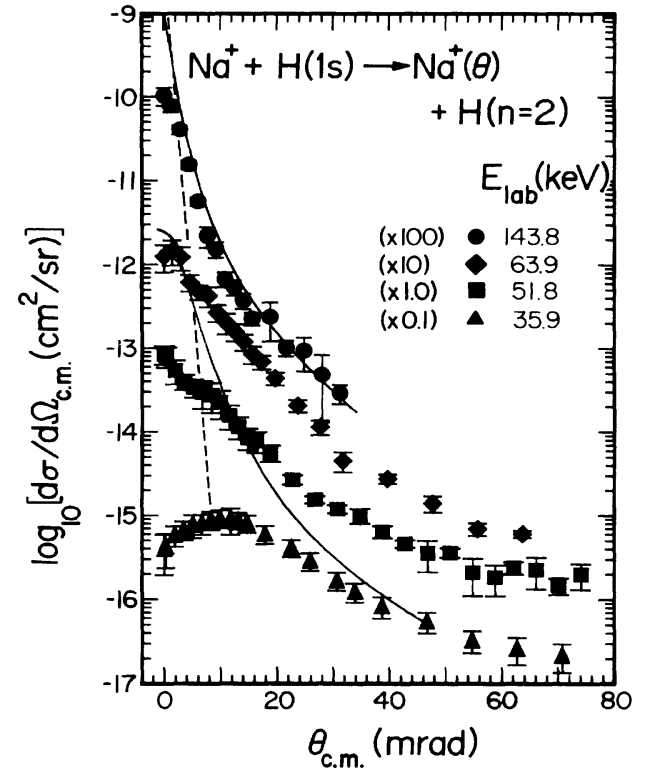

FIG. 2. $\mathrm{Na}^{+}+\mathrm{H}$ ADCS's. The upper and lower solid lines are the predictions of the screened eikonal theory for 143.8 and $63.9 \mathrm{keV}$, respectively. The screened first Born calculation for $143.8 \mathrm{keV}$ is shown with a dashed line.

tude larger than the $\mathrm{NaH}^{+}$cross sections at equivalent angles and velocities. By numerical integration of the ADCS's, we have obtained values for the total excitation cross sections as a function of energy. These are shown in Fig. 3. The $\mathrm{MgHe}^{+}$cross sections are typically $2-10$ times greater than the $\mathrm{NaH}^{+}$cross sections at the same projectile velocity.

The different behavior of the two QOE systems can be understood by considering the potential curves associated with their respective transient molecular states. These are shown schematically in Fig. 4. ${ }^{7,15,16}$ In the separated atom limit, the molecular states corresponding to the ground and first excited states of $\mathrm{Mg}^{+}$are separated by $4.4 \mathrm{eV}$. While radial coupling between these states exhibits a broad maximum at $\sim 3$ a.u., ${ }^{15}$ there are no strongly avoided crossings until $r \simeq 1$ a.u. At this point, rapid promotion of the He ground-state orbitals to the $3 d \sigma$ united atom orbitals results in strong radial and rotational coupling between the states correlating with the ground and excited states of $\mathrm{Mg}^{+}$and states associated with charge transfer and $\mathrm{He}$ excitation channels. ${ }^{7}$ The $\mathrm{NaH}^{+}$curves are qualitatively different. Because of their large energy difference in the separated atom limit, coupling between the $\mathrm{H}(1 s)$ and $\mathrm{H}(2 p)$ states is very small until about $r=2.5$ a.u., when nuclear repulsion begins to dominate the molecular orbital (MO) energies. ${ }^{15,16}$ The large $\Delta E$ of these states in the separated atom limit results in a particularly strong "running together" of the energy levels for $r \lesssim 2.5$ a.u., with consequent large values of radial and rotational coupling matrix elements. ${ }^{15}$

For low-energy $\mathrm{MgHe}^{+}$collisions, large-r coupling (the "direct" mechanism) is ineffective, and all excitation must take place via "molecular" transitions at $\sim 1$ a.u. ${ }^{7,17}$ As 


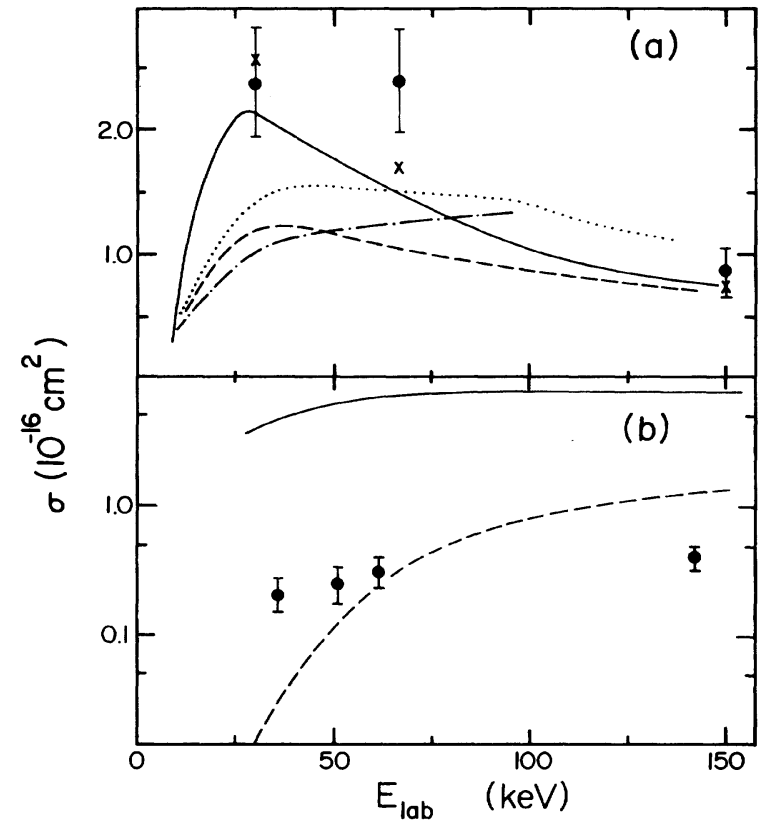

FIG. 3. Total excitation cross section vs projectile energy (note change from logarithmic to linear scales): (a) $\mathrm{Mg}^{+}+\mathrm{He}$; $(\bullet)$ present work; $(\ldots$.$) , emission cross sections from Ref. 3;$ (..-), total cross sections, corrected for cascading, with $40 \%$ quoted error, Ref. 3; (-), three-state AO CC calculation, with Hartree-Fock core potential, Ref. 8; (-..), molecular state CC calculation, Ref. 6; $(X)$, recent seven-state AO CC results of ND, Ref. 19. (b) $\mathrm{Na}^{+}+\mathrm{H}$; (๑), present work; (-) and (---), first Born and screened eikonal calculations, respectively, of this work. Error bars represent the SDM of all angleintegrated runs, with target thickness and solid-angle errors combined in quadrature.

the energy is increased, large-r coupling becomes more effective, resulting in a peak in the total cross section at the Massey maximum of $=30 \mathrm{keV}$. In the $\mathrm{NaH}^{+}$case, $\mathrm{H}(1 s)-\mathrm{H}(2 p)$ coupling is so weak at large $r$, due to the large energy separation, that "direct" excitation is dominated at all energies by "molecular" transitions at $r \lesssim 2$ a.u. Nonetheless, we do see the onset of direct excitation in the rapidly rising values of $d \sigma(\theta=0) / d \Omega$ with $E$. The dramatic rise ( $2 \frac{1}{2}$ orders of magnitude) with energy of the small angle $\mathrm{NaH}^{+}$ADCS's does not produce a corresponding rise in the total cross section; this is because the major contribution to the total comes from the angular regions where the product $\theta d \sigma / d \Omega(\theta)$ is a maximum.

Given the relatively large energy separation $\Delta E$ in $\mathrm{H}$, and the fact that the exciting closed shell is charged (which should correspond to a higher value of $a$ in the Massey scaling formula than would a neutral closed core), it is not surprising that while the $\mathrm{MgHe}^{+}$system is well past the Massey peak for collision velocities of 0.5 a.u., the $\mathrm{NaH}^{+}$total cross sections are still increasing. The velocity regime below the Massey peak is just where molecular excitation is expected to play a significant role. Indeed, the nonzero maximum of the $\mathrm{NaH}^{+}$ADCS's at $35.9 \mathrm{keV}$ is characteristic of the molecular excitation-

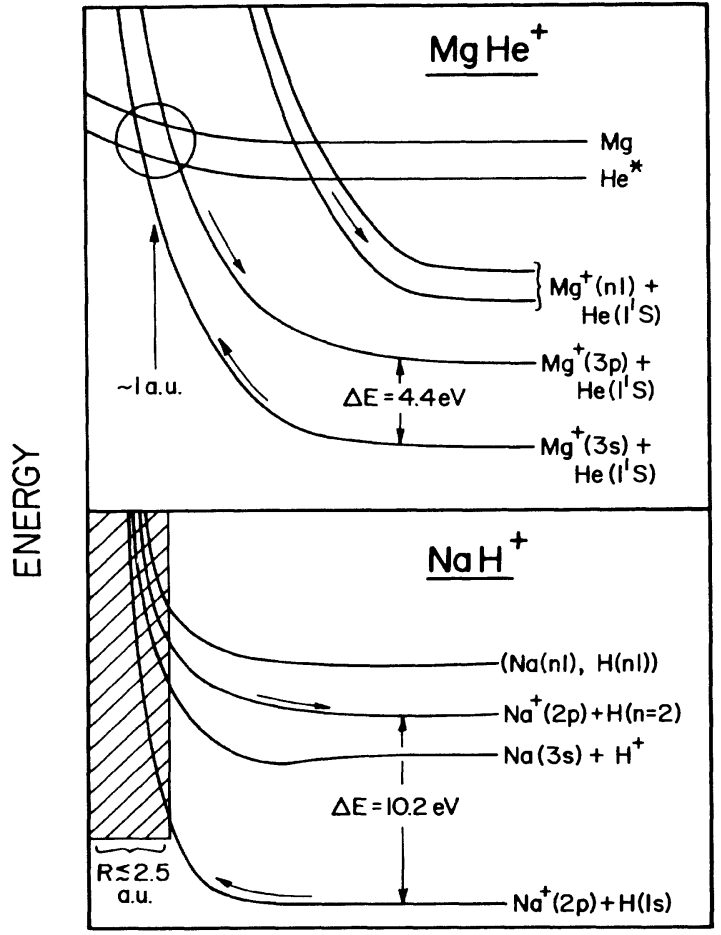

INTERNUCLEAR SEPARATION, R

FIG. 4. Schematic representations of $\mathrm{MgHe}^{+}$and $\mathrm{NaH}^{+}$ quasimolecules. The value of $\simeq 1$ a.u. for the crossing of the $\mathrm{Mg}^{+}$ground state with higher-lying channels has been calculated by Courbin-Gaussorgues and co-workers for the $\mathrm{NaHe}$ isoelectronic system (Ref. 7).

dominated ADCS's seen at considerably lower collision velocities in rare-gas core QOE systems. ${ }^{18}$

The only theoretical treatments of these collisions to date have been the extensive CC calculations of Nielsen and Dahler (ND) for $\mathrm{MgHe}^{+}$collisions. ${ }^{1,8,19}$ We have performed screened first Born and screened eikonal approximation calculations for comparison with our $\mathrm{NaH}^{+}$ data. ${ }^{20}$ The $\mathrm{CC}$ calculations predict excitation probability $P$ as a function of impact parameter $b$ and must be transformed, using an appropriate scattering potential, to yield values of $d \sigma(\theta) / d \Omega$ (Fig. 1). We have used an exponential scattering potential ${ }^{18} V(R)=A \exp (-B R)$ with the strength and range parameters $A=46$ a.u. and $B=2.88 \quad$ (a.u. $^{-1}$ derived from Sondergaard and Mason. ${ }^{21}$ The screened Born and eikonal calculations are directly comparable to our data and are shown in Fig. 2. The agreement of the CC calculations with our measurements is very good, given the ambiguity introduced by the $b-\theta$ transform. The AO basis used in these calculations is of course consonant with direct excitation. Clearly, the theory is handling the valence-electron-core interaction adequately. A significant divergence from the ND theory occurs, however, at 66.7 and $150 \mathrm{keV}$ at center-of-mass angles of about $17 \mathrm{mrad}(9.3 \mathrm{keV} \mathrm{deg})$ and $7 \mathrm{mrad}(8.6$ $\mathrm{keV} \mathrm{deg}$ ), respectively. This divergence, corresponding to an impact parameter of about 1.0 a.u., is believed to be due to the onset of significant molecular excitation pro- 
cesses at the strongly avoided $1-a . u$. curve crossings. In a limited series of data runs, enhancements of He excitation and charge transfer were observed at these large angles. ${ }^{22}$ One would not expect the ND theory to handle this "molecular" mechanism properly, since it is an AO theory. (The data at $30 \mathrm{keV}$ taken at the largest angles do not correspond to a small enough impact parameter for this divergence to be seen.) Another possible cause for the deviation between our measurements and the ND theory at large angle is that the $b-\theta$ transform may begin to lose validity at the smallest impact parameters.

Our screened first Born and eikonal calculations for the $\mathrm{NaH}^{+}$collisions are obviously inadequate, although the large angle data at $143.8 \mathrm{keV}$ is fitted fairly well with the eikonal theory. This agreement is presumably fortuitous, in that perturbative theories should break down first at small $b$. We expect that close-coupling calculations with a molecular basis will be necessary to reproduce these data. As mentioned earlier, the simplified electron-core interaction and perfectly known target states should simplify such calculations.

The $\mathrm{MgHe}^{+}$integrated total cross sections [Fig. 3(a)] are in good agreement with the most recent ND calculations, but are in significant disagreement with the MO close-coupling calculation of Knopfle and $\mathrm{Kempter}^{6}$ and the optical measurements of Andersen, Andersen, and Jensen, ${ }^{3}$ which have quoted statistical plus systematic errors of $40 \%$. We note that the uncertainty associated with the $b-\theta$ transformation procedure is eliminated in comparing experimental and theoretical total cross sections.

In QOE excitation collisions, a critical parameter is thus seen to be the energy separation $\Delta E$ between the ground state and excited state of the active electron. Molecular excitation, which has been regarded as a lowenergy (few keV) phenomenon in valence-shell excitation, can, in fact, dominate direct excitation if $\Delta E$ is sufficiently large. In the $\mathrm{MgHe}^{+}$system, there is a smooth transfer from essentially complete molecular excitation at $3 \mathrm{keV}$ (Ref. 17) to primarily direct excitation at $150 \mathrm{keV}$; with $\mathrm{NaH}^{+}$, the two processes are of comparable importance at this energy. The relatively large $\Delta E$ in $\mathrm{H}$ delays the shift from one excitation mechanism to the other.

We wish to acknowledge helpful discussions with Svend Eric Nielsen, Nils Andersen, John Wm. Edwards, and especially Ron Olson. We also thank C. A. McWhorter for much careful instrument making. This work has been supported by National Science Foundation Grant No. PHY-8406552.
*Present address: Engineering Development Laboratory, E. I. duPont Co., 101 Beech St., Wilmington, DE 19898.

${ }^{1}$ N. Andersen and S. E. Nielsen, Adv. At. Mol. Phys. 18, 265 (1982), and references therein.

${ }^{2}$ H. S. W. Massey, Rep. Prog. Phys. 12, 248 (1949).

${ }^{3}$ N. Andersen, T. Andersen, and K. Jensen, J. Phys. B 9, 1373 (1976).

${ }^{4}$ See, e.g., J. O. Olsen, N. Andersen, and T. Andersen, J. Phys. B 10, 1723 (1977).

${ }^{5}$ See, e.g., E. Horsdal Pedersen et al., J. Phys. B 11, L317 (1978); W. Heydenreich et al., Z. Phys. A 312, 285 (1983).

${ }^{6}$ W. Knopfle and V. Kempter, Z. Phys. A 314, 283 (1983).

${ }^{7}$ C. Courbin-Gaussorgues, V. Sidis, and J. Vaaben, J. Phys. B 16, 2817 (1983); C. Courbin-Gaussorgues and V. Sidis, ibid. 18,699 (1985).

${ }^{8}$ See, e.g., S. E. Nielsen and J. S. Dahler, Phys. Rev. A 16, 563 (1977), and references therein.

${ }^{9}$ See, e.g., M. Kimura and J. Pascale, J. Phys. B 18, 2719 (1985), and references therein.

${ }^{10}$ See, e.g., F. Aumayr, G. Lakits, and H. Winter, J. Phys. B 20 , 2025 (1987), and references therein.

${ }^{11}$ See, e.g., R. J. Allan, J. Phys. B 19, 321 (1986), and references therein.
12 J. T. Park, Adv. At. Mol. Phys. 19, 67 (1983).

${ }^{13}$ J. T. Park et al., Rev. Sci. Instrum. 54, 1247 (1983).

${ }^{14} \mathrm{E}$. Redd, Ph.D. thesis, University of Missouri-Rolla, 1986 (unpublished).

${ }^{15}$ R. E. Olson (private communication).

${ }^{16}$ M. Kimura, R. E. Olson, and J. Pascale, Phys. Rev. A 26, 3113 (1982).

${ }^{17}$ N. Andersen et al., J. Phys. B 12, 2541 (1979).

${ }^{18}$ See, e.g., J. O. Olsen, K. Vedel, and P. Dahl, J. Phys. B 12 , 929 (1979); J. Fayeton, N. Anderson, and M. Barat, ibid. 9, L149 (1976).

${ }^{19}$ S. E. Nielsen and J. S. Dahler (private communication)

${ }^{20}$ J. L. Peacher et al., Phys. Rev. A 30, 729 (1984).

${ }^{21}$ N. A. Sondergaard and E. A. Mason, J. Chem. Phys. 62, 1299 (1975). The transformation is made using the classical formula for scattering,

$$
b P(b)=-[d \sigma(\theta) / d \Omega] \sin (\theta) d \theta / d b,
$$

where $\theta=A B b K_{0}(B b) / E$ for the Born-Meyer potential, and $K_{0}$ is the modified Bessel function of the second kind.

${ }^{22}$ E. Redd et al., Nucl. Instrum. Methods Phys. Res. Sect. B 24/25, 305 (1987). 